\title{
Optimization of the Alpha Energy Deposited in Radioluminescence Thin Film for Alphaphotovoltaic Application
}

\author{
Dessy Purbandari $^{1^{*}}$, Ferdiansjah $^{1}$, Tjipto Sujitno ${ }^{2}$ \\ ${ }^{1}$ Nuclear Engineering and Engineering Physics Department, Universitas Gadjah Mada ${ }^{2}$ National Nuclear Energy Agency of Indonesia \\ Jl. Grafika 2 Yogyakarta 55281, Indonesia. Tel. +62-274-580882, Fax. +62-274-580882 \\ "Email: dessy.purbandari@mail.ugm.ac.id
}

\begin{abstract}
Activated zinc sulfide ( $\mathrm{ZnS})$ is a semiconductor material which able to emit photon in the form of visible light when expose to external energy. The capability of activated $\mathrm{ZnS}$, mainly doped with silver $(\mathrm{Ag})$ and copper $(\mathrm{Cu})$, to convert radiation become light to make it potentially applicable as the radioluminescent thin film for alphaphotovoltaic-type nuclear battery. One of the important specifications of the radioluminescence layer that influences the fluorescence efficiency is the thickness. This work presents a study on the thickness optimization for $\mathrm{ZnS}: \mathrm{Ag}: \mathrm{Cu}$ as the radioluminescent film for alpha particles using Monte Carlo model. Simulation to study alpha particles' energy deposited by using Stopping and Range of Ions in Matter/TRansport of Ions in Matter (SRIM/TRIM) code. The model examined the transport of $5.485 \mathrm{MeV}$ alpha particles emitted by ${ }^{241} \mathrm{Am}$ to determine the best thickness based on energy deposition depth. Based on TRIM module simulation, the optimal thickness for radioluminescence film is approximately 19-22 $\mu$ m. Most energy from 5.485 MeV alpha particles is deposited in $18.92 \mu \mathrm{m}$ depth activated zinc sulfide. The results from SRIM/TRIM model then compare with analytical calculation using Bragg-Kleman rule. The alpha particles stop at $22 \mu \mathrm{m}$ from the SRIM/TRIM simulation while using Bragg-Kleman formula the alpha particles stop at $23.51 \mu \mathrm{m}$.
\end{abstract}

Keywords: Alphaphotovoltaic, Radioluminescence, SRIM/TRIM, ZnS:Ag:Cu

Abbreviations: Stopping and Range of Ions in Matter (SRIM), TRansport of Ions in Matter (TRIM), zinc sulfide (ZnS)

\section{INTRODUCTION}

Zinc sulfide $(\mathrm{ZnS})$ is a direct gap II-VI intrinsic semiconductor material which abundant and environmental friendly. $\mathrm{ZnS}$ material has a wide band gap of $3.66 \mathrm{eV}$, good chemical stability, high light transmittance and a low dispersion in the visible and infrared region. An extensive research effort about $\mathrm{ZnS}$ material has developed rapidly due to its potential utilization for numerous fields such as in cathode ray tube, semiconductor light-emitting diode, and bio lightemitting diode. In addition, $\mathrm{ZnS}$ material is suitable for use as the radioluminescence layer in an alphaphotovoltaic indirect-conversion nuclear battery (Russo et al., 2017).

Nuclear battery has potential for use as the substitute of conventional battery due to radioisotopes possess theoretical energy densities 1000 times greater than chemical battery, longer operation time, and durability in extreme environments. Nuclear battery with power scale from $\mu \mathrm{W}$ until $\mathrm{mW}$ offers promising alternative as power supply for sensor and low power electronic devices. Nuclear battery can supply sufficient and long-lived power for various applications such as mobile sensor platforms, military and remote electronic devices.

Radioluminescence layer in an alphaphotovoltaic indirect-conversion nuclear battery absorbs alpha particles emitted from the radioisotope source and convert the radiation in the form of visible light. The visible light is absorbed and converted into electricity by photovoltaic which is placed adjacent to the radioluminescence layer. The characteristic of the radioluminescence layer plays important role to absorb the energy from radiation and transport the emitted luminescence produce by the radioluminescence layer. Appropriate thickness of the radioluminescence layer is needed to optimize radiation absorption into luminescence. Design proper structure of phosphor or radioluminescence layer may increase the luminescence efficiency both production luminescence and transmission the emitted luminescence in the material (Xu et al., 2014).

Xu et al. (2014) have researched about the effect of phosphor layer structure to radioluminescence intensity. The research is examined phosphor layer with different thickness and measured the radioluminescence intensity by using Cary Eclipse fluorescence spectrophotometer (Agilent Technologies, USA) with $4.93 \mathrm{mCi} / \mathrm{cm}^{2}{ }^{63} \mathrm{Ni}$ and $2.88 \mathrm{mCi} / \mathrm{cm}^{2}{ }^{147} \mathrm{Pm}$ beta particle sources. Based on the radioluminescence measurements, it is apparent that radioluminescence intensity has correlation with the phosphor structure. The phosphor structure has influence on RL intensity in two factors: absorption of the radiation and transmission of the luminescence in the phosphor layer. If the phosphor layer is thicker than the penetration depth of the radiation, the negative effect of luminescence self-absorption in the phosphor layer was bigger than the increasing of energy deposited. On the contrary, only a few energy from alpha particles will be deposited in the phosphor layer if the layer is thinner 
than the penetration depth. Thus, research about the optimal layer thickness for indirect-conversion nuclear battery is important to do.

Stopping and Range of Ions in Matter/TRansport of Ions in Matter (SRIM/TRIM) code were used to determine optimum thickness based on energy deposition depth by alpha particles in the $\mathrm{ZnS}$ material. The model examined the radiation of $5.485 \mathrm{MeV}$ alpha particles emitted by Americium-241 $\left({ }^{241} \mathrm{Am}\right)$. Energy deposition in $22 \mu \mathrm{m}$ thickness of $\mathrm{ZnS}: \mathrm{Ag}: \mathrm{Cu}$ was calculated for slab geometry using SRIM/TRIM. These geometry is the most possible geometry deployed for alphaphotovoltaic indirect-conversion nuclear battery (Oh, 2011).

The results from software simulation are compared to theoretical calculation using Bragg-Kleman rule. Formula from the Bragg-Kleman rule can be found in Knoll (1989). This formula provides information about the range of particles in a material without energy loss data. Bragg-Kleman rule calculated the range of certain particles in a material using range information of the particles in another material. Bragg-Kleman rule is shown as follows:

$$
\frac{R_{1}}{R_{2}}=\frac{\rho_{2}}{\rho_{1}} \sqrt{\frac{A_{1}}{A_{2}}}
$$

Where $\rho$ is the density of the material, A is the atomic weight or effective atomic weight if it is a compound material, R is the particles' range. Theoretical calculation about particles' range in a certain material using Bragg-Kleman law exceeds two-step process:

1. Determine the range in air;

$$
\mathrm{R}(\mathrm{mm})=(0,05 \mathrm{~T}+2,85) T^{\frac{\pi}{2}} 4<\mathrm{T} \leq 15 \mathrm{MeV}
$$

Symbol R denoting particles' range in the air, whereas $\mathrm{T}$ shows particles' energy emitted by the source.

2. The data obtained from process number 1 is used to determine the range in the material by using BraggKleman rule.

\section{MATERIALS AND METHODS}

This research is conducted by using theoretical computation and analytical calculation. Initial literature study is done to find characteristic data of alpha radiation source Americium-241 $\left({ }^{241} \mathrm{Am}\right)$ and information about radioluminescence material $\mathrm{ZnS}: \mathrm{Ag}: \mathrm{Cu}$. The selection of ${ }^{241} \mathrm{Am}$ as isotope to use in a nuclear battery based on its long half-life, high purity and energy density, short endpoint energy. Research about nuclear battery is focused on alpha and beta radiation due to their relatively short range, thus their application would not require large-scale configuration.

\section{Simulation and Analytical Calculation}

Sets of personal computer with Windows 10 operating system and Core i3-2328M $2.20 \mathrm{GHz}$ is used to run software SRIM 2013. Actually, there are few codes suitable for modeling alpha particle interactions. The code chosen in this study which treat alpha particle interaction with matter is SRIM/TRIM 2013. SRIM/TRIM 2013 demonstrates mono-directional and mono-energetic alpha particles interact with matter in only the slab geometry.

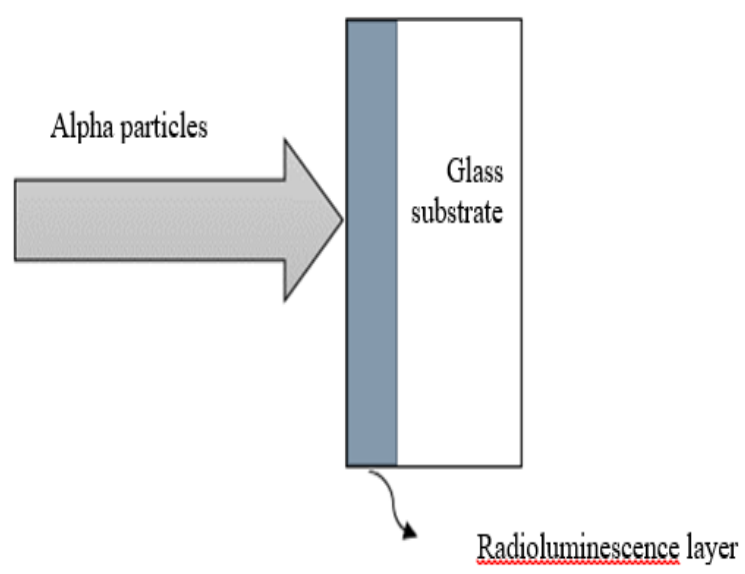

Figure 1. Slab model used for SRIM/TRIM simulation. A monodirectional and mono-energetic alpha beam impinges on the slab target.

Results obtained from SRIM/TRIM simulation are compared to analytical calculation using Bragg-Kleman rule. Alpha particle range in $\mathrm{ZnS}$ material is calculated using Equation (1) and (2).

\section{RESULTS AND DISCUSSION}

\section{SRIM/TRIM Simulation}

Simulation is done using 99,999 ions for increasing accuracy of the calculation. The accuracy of calculation is determined by the number of ions followed. Typically, a calculation for 1000 ions will give better than $10 \%$ accuracy (SRIM, 2010). Thus, simulation with 99,999 ions can achieve the accuracy for more than $90 \%$.

Figure 2 illustrates the tracks of alpha particles within the slab and demonstrates their relatively nearly linear paths. When a charged particle penetrates in matter, in this case is alpha particle which has positive charged, a charged particle will interact with the electrons and nuclei present in the material through the electromagnetic force. For particles with energy more than $1 \mathrm{MeV}$, the charge particles will mostly interact with the electrons present in the material. However there are some of the ions collide with the nucleus which make the direction change. Those interactions are showed in Figure 2, the scattered ions are the ions collide with the nucleus in the $\mathrm{ZnS}$ material. 


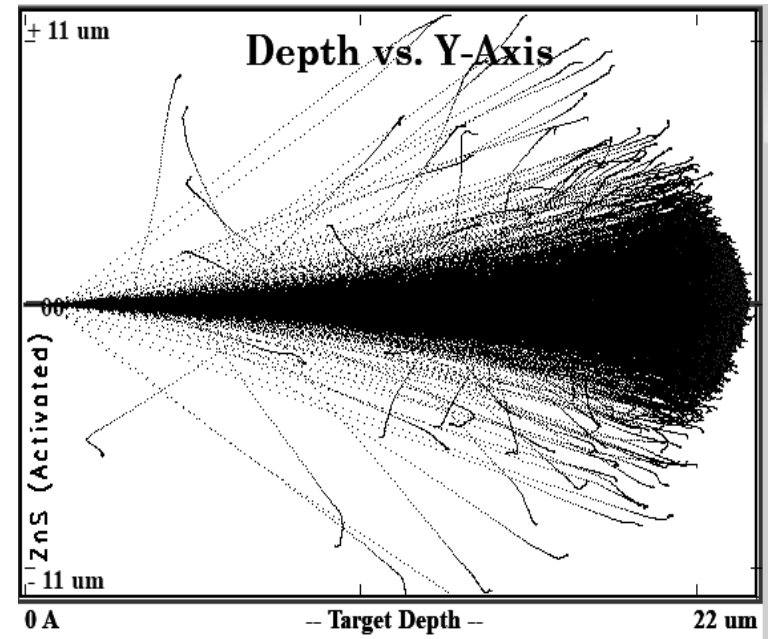

Figure 2. Illustration the tracks of Am-241 alpha particles within the ZnS slab using SRIM/TRIM.

Figure 3 shows the alpha particle's Bragg curve in the $\mathrm{ZnS}$ target. The SRIM/TRIM result from the $\mathrm{ZnS}$ slab model shows that the peak of the Bragg curve occurs at around $18.92 \mu \mathrm{m}$. The energy loss maximum of charged particles near the end of the range called as "Bragg peak". Beyond the Bragg peak, the energy deposition drops sharply. This is because alpha particles have lost almost all of their energy when they traveled at the range $18.92 \mu \mathrm{m}$. As the particle penetrates in the medium, its energy loss per unit length will change. The energy loss of a particle as a function of its distance of penetration is shown in Table 1. The Bragg curve shows that alpha particles have lost all of their energy in the $\mathrm{ZnS}$ material at around $22 \mu \mathrm{m}$.

Energy deposition depth of alpha particles is shown in Table 1. The percentage of energy deposited can be calculated by adding up all the energy loss from alpha particles when penetrates in matter. In addition, Table 1 shows that the maximum energy deposition occurs between 18 to $19 \mu \mathrm{m}$ from the source. SRIM/TRIM predicts the percentage of energy deposited in this layer is $\sim 7.24 \%$ for slab configuration.

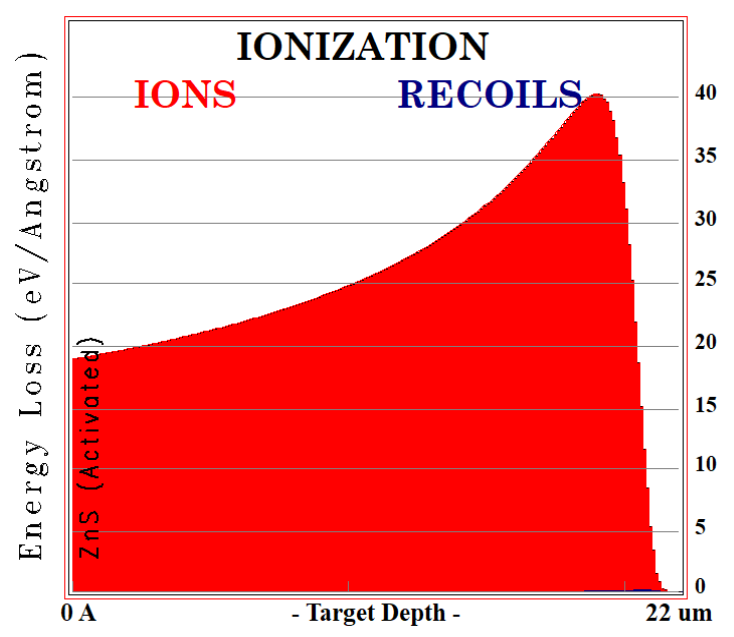

Figure 3. The alpha particle's Bragg curves at in $\mathrm{ZnS}$ target using SRIM/TRIM.
When a charged particle penetrates in matter, it will loss its energy by transferring a small amount of energy to a large number of electrons along its trajectory. Based on Table 1, alpha radiation deposited $99.74 \%$ of their energy into the atom target. The main interaction which cause the alpha particles loss their energy is excitation and electron ionization. The type of energy loss due to interaction of the charged particle with electrons is often called as "energy loss due to ionization". When a charged particle has sufficient energy to excite or ionize atoms in the medium, it will deposit several eV of its energy until it lost all the kinetic energy and come to rest. The term "energy loss due to ionization" stated all the energy loss from both ionization interaction and electron excitation, since many atoms are only brought to an excite state.

Alpha particles mostly deposited all of their energy between 18 until $19 \mu \mathrm{m}$ in the $\mathrm{ZnS}$ target. The percentage of energy deposited in this layer is $\sim 7.24 \%$ which is the Bragg peak of the curve. Beyond this distance, the energy deposited drops sharply from $\sim 6.69 \%$ at the range 19 until $20 \mu \mathrm{m} ; 2,56 \%$ at the range 20 until $21 \mu \mathrm{m} ; 0,04 \%$ at the range 21 until $22 \mu \mathrm{m}$. Based on SRIM/TRIM simulation we can conclude that theoretical thickness needed for radioluminescence layer can optimally convert all the radiation energy from ${ }^{241} \mathrm{Am}$ is approximately 19 until $22 \mu \mathrm{m}$.

Table 1. SRIM/TRIM calculations for predicting energy deposition in $\mathrm{ZnS}$ target for slab model.

\begin{tabular}{lll}
\hline Range $(\boldsymbol{\mu m})$ & Energy $(\mathbf{k e V})$ & \% deposited \\
\hline $0-1$ & 189,8 & 3,46 \\
$1-2$ & 193,9 & 3,54 \\
$2-3$ & 198,3 & 3,62 \\
$3-4$ & 203,2 & 3,70 \\
$4-5$ & 208,5 & 3,80 \\
$5-6$ & 214,2 & 3,91 \\
$6-7$ & 220,4 & 4,02 \\
$7-8$ & 227,4 & 4,15 \\
$8-9$ & 234,9 & 4,28 \\
$9-10$ & 243,3 & 4,44 \\
$10-11$ & 252,5 & 4,60 \\
$11-12$ & 263,3 & 4,80 \\
$12-13$ & 275,2 & 5,02 \\
$13-14$ & 289,2 & 5,27 \\
$14-15$ & 305,4 & 5,57 \\
$15-16$ & 324,5 & 5,92 \\
$16-17$ & 347,1 & 6,33 \\
$17-18$ & 373,1 & 6,80 \\
$18-19$ & 397,1 & 7,24 \\
$19-20$ & 366,9 & 6,69 \\
$20-21$ & 140,6 & 2,56 \\
$21-22$ & 2,3 & 0,04 \\
Total & $\mathbf{5 4 7 1}$ & $\mathbf{9 9 , 7 4}$ \\
\hline
\end{tabular}

\section{Theoretical Calculation}

The range of alpha particles in air with normal temperature and pressure in the energy range $4<\mathrm{E}<$ $15 \mathrm{MeV}$ is calculated by using an empirical equation: 
$\mathrm{R}(\mathrm{mm})=(0.05 \mathrm{~T}+2.85) \mathrm{T}^{3 / 2} \quad 4<\mathrm{T} \leq 15 \mathrm{MeV}$

Alpha particles emitted from ${ }^{241} \mathrm{Am}$ decay process have energy of $5.485 \mathrm{MeV}$.

$\mathrm{R}(\mathrm{mm})=(0.05 \times 5.485+2.85) 5.485^{3 / 2}$

$\mathrm{R}(\mathrm{mm})=40,134 \mathrm{~mm}$

Bragg-Kleman rule to compute the range of alpha particles in any material is using the following equation:

$\mathrm{R}_{1} / \mathrm{R}_{2}=\left(\rho_{1} / \rho_{2}\right)\left(\mathrm{A}_{1} / \mathrm{A}_{2}\right)^{1 / 2}$

Because $\mathrm{ZnS}$ is in the form of compound material, so that atomic weight $A_{i}$ is calculated using effective atomic weight $A_{\text {eff }}$ :

$$
\sqrt{A_{e f}}=\left(\sum_{i=1}^{n} \frac{w_{i}}{\sqrt{A_{i}}}\right)
$$

Air is consisted by $20 \%$ oxygen and $80 \%$ nitrogen, so that for air:

$$
\begin{gathered}
w_{o}=\frac{0,2 \times M_{0}}{0,2 \times M_{0}+0,8 \times M_{N}}=\frac{0,2 \times 16}{0,2 \times 16+0,8 \times 14}=0.222 \\
w_{N}=1-0.222=0.778 \\
\sqrt{A_{e f}}=\left(\frac{w_{0}}{\sqrt{A_{O}}}+\frac{w_{N}}{\sqrt{A_{N}}}\right)^{-1}=\left(\frac{0,222}{\sqrt{16}}+\frac{0,778}{\sqrt{14}}\right)^{-1}=3.796
\end{gathered}
$$

$\mathrm{A}_{\text {ef,air }}=(3.796)^{2}=14.41$

For $\mathrm{ZnS}$ material is calculated by using the following formula:

$$
\begin{aligned}
& w_{Z n}=\frac{M_{Z n}}{M_{Z n}+M_{S}}=\frac{65}{65+32}=0.67 \\
& w_{N}=1-0.67=0.33 \\
& \sqrt{A_{\text {ef }}}=\left(\frac{w_{Z n}}{\sqrt{A_{Z n}}}+\frac{w_{S}}{\sqrt{A_{S}}}\right)^{-1}=\left(\frac{0,67}{\sqrt{65}}+\frac{0,33}{\sqrt{32}}\right)^{-1}=7.07 \\
& \mathrm{~A}_{\text {ef,ZnS }}=(7.07)^{2}=49.99 \\
& \mathrm{Bragg}_{\mathrm{K}} \mathrm{Kleman} \text { rule: } \\
& \mathrm{R}_{\mathrm{ZnS}}=\left(\rho_{\mathrm{ZnS}} / \rho_{\mathrm{udara}}\right)\left(\mathrm{A}_{\mathrm{ZnS}} / \mathrm{A}_{\mathrm{udara}}\right)^{1 / 2} \\
& =(0.00129 / 4.1) \times(49.99 / 14.41)^{1 / 2}=5.86 \times 10^{-4} \\
& \mathrm{R}_{\mathrm{ZnS}}=5.86 \times 10^{-4} \times \mathrm{R}_{\mathrm{udara}} \\
& \mathrm{R}_{\mathrm{ZnS}}=5.86 \times 10^{-4} \times 40.134=23.51 \mu \mathrm{m} .
\end{aligned}
$$

The range of alpha particles have energy of $5.485 \mathrm{MeV}$ in $\mathrm{ZnS}$ material is $23.51 \mu \mathrm{m}$.

\section{CONCLUSIONS}

Based on the study it can concluded the SRIM/TRIM result from the $\mathrm{ZnS}$ slab model shows that the peak of the Bragg curve occurs at around $18.92 \mu \mathrm{m}$. The Bragg curve shows that alpha particles have lost all of their energy in the $\mathrm{ZnS}$ material at around $22 \mu \mathrm{m}$. Alpha radiation deposited $99.74 \%$ of their energy into the atom target. The main interaction which cause the alpha particles loss their energy is excitation and electron ionization.

Alpha particles mostly deposited all of their energy between 18 until $19 \mu \mathrm{m}$ in the $\mathrm{ZnS}$ target. The percentage of energy deposited in this layer is $\sim 7.24 \%$ which is the Bragg peak of the curve. Beyond this distance, the energy deposited drops sharply from $\sim 6.69 \%$ at the range 19 until $20 \mu \mathrm{m} ; 2,56 \%$ at the range 20 until $21 \mu \mathrm{m} ; 0,04 \%$ at the range 21 until $22 \mu \mathrm{m}$. Based on SRIM/TRIM simulation we can conclude that theoretical thickness needed for radioluminescence layer can optimally convert all the radiation energy from ${ }^{241} \mathrm{Am}$ is approximately 19 until $22 \mu \mathrm{m}$. The alpha particles stop at $22 \mu \mathrm{m}$ from the SRIM/TRIM simulation while using Bragg-Kleman formula the alpha particles stop at $23.51 \mu \mathrm{m}$.

\section{ACKNOWLEDGEMENTS}

Author would like to gratefully thank PSTA BATAN Yogyakarta and PT Ensterna for the opportunity of doing research in the institution.

\section{REFERENCES}

Hong L, Tang XB, Xu ZH. 2014. Radioluminescent Nuclear Batteries with Different Phosphor Layers. Nuclear Instrument and Methods in Physics Research 334:112-118.

Oh K. 2011. Modeling and Maximum Theoritical Efficiencies of Linearly Graded Alphavoltaic and Betavoltaic Cells. [Dissertation]. Faculty of Graduate School, University of Missouri. [Columbia]

Sulistya E. 2016. Penentuan Dosis Optimum pada Radioterapi Proton dengan Menggunakan Program SRIM. [Dissertation]. Gadjah Mada University, Yogyakarta. [Indonesian]

Sychov M, Kavetsky A, Yakubova G. 2008. Alpha Indirect Conversion Radioisotope Power Source. Applied Radiation and Isotopes 66(2):173-177.

Xu ZH, Tang XB, Hong L. 2015. Structural Effects of ZnS:Cu Phosphor Layers on Beta Radioluminescence Nuclear Battery. Journal Radioanal Nuclear Chemistr, 303:2313-2320.

Ziegler J, Biersack J, Ziegler M. 2008. SRIM-The Stopping and Range of Ions in Matter. SRIM Co., Chester, Maryland. 